

\title{
An Euler-Poincaré bound for equicharacteristic étale sheaves
}

\author{
Carl A. Miller
}

\begin{abstract}
The Grothendieck-Ogg-Shafarevich formula expresses the Euler characteristic of an étale sheaf on a characteristic- $p$ curve in terms of local data. The purpose of this paper is to prove an equicharacteristic version of this formula (a bound, rather than an equality). This follows work of R. Pink.

The basis for the proof of this result is the characteristic- $p$ Riemann-Hilbert correspondence, which is a functorial relationship between two different types of sheaves on a characteristic- $p$ scheme. In the paper we prove a one-dimensional version of this correspondence, considering both local and global settings.
\end{abstract}

\section{Introduction}

Let $k$ be an algebraically closed field of characteristic $p>0$. Let $Y$ be a smooth projective $k$-curve, and let $N$ be a constructible étale sheaf of $\mathbb{F}_{p}$-vector spaces on $Y$. We are concerned with computing the sizes of cohomology groups of the pair $(Y, N)$.

The following theorem (the Grothendieck-Ogg-Shafarevich formula) provides a starting point. For any étale $\mathbb{F}_{\ell}$-sheaf $M$ on $Y$, where $\ell$ denotes a prime different from $p$, let $\chi(Y, M)$ denote the Euler characteristic of $M$. (That is, $\chi(Y, M)=$ $\sum_{i=0}^{2}(-1)^{i} \operatorname{dim}_{\mathbb{F}_{\ell}} H^{i}(Y, M)$.)

Theorem 1.0.1. Let $\ell$ be a prime which is not equal to $p$. Let $G$ be a constructible $\mathbb{F}_{\ell}$-sheaf on $Y$ such that:

(1) $G$ is locally constant on some open subcurve $Y^{\prime} \subseteq Y$, and

(2) $G_{y}=\{0\}$ for all points $y \in Y \backslash Y^{\prime}$.

Then,

$$
\chi(Y, G)=(2-2 g) m-\sum_{y \in Y \backslash Y^{\prime}}\left(m+\operatorname{Sw}_{y}(G)\right),
$$

MSC2000: primary 14F20; secondary 13A35, 14F30.

Keywords: characteristic- $p$ curves, Grothendieck-Ogg-Shafarevich formula, étale sheaves,

Riemann-Hilbert correspondence, Frobenius endomorphism, minimal roots.

The author was supported by NSF grant DMS-0502170 during the publication of this paper. 
where $g$ denotes the genus of $Y$ and $m=\operatorname{rank} G$.

In Equation (1.0.2), $\mathrm{Sw}_{y}(G)$ denotes a local invariant called the "Swan conductor." (See [Raynaud 1995] for a discussion of this formula.)

It is desirable to have a similar formula for the Euler characteristic of an $\mathbb{F}_{p}$-sheaf on $Y$. Unfortunately the equicharacteristic case presents some difficulties. It is possible to construct two $\mathbb{F}_{p}$-sheaves $N_{1}$ and $N_{2}$ on the same curve $Y$, both sharing the same rank and the same local data but possessing different Euler characteristics. (See Example 3.2.9.)

So an exact analogue of Theorem 1.0.1 is not possible. The purpose of this paper is to prove a lower bound for $\chi(Y, N)$ which matches the form of (1.0.2). This builds on previous work of R. Pink [2000].

The proof of the lower bound is based on the relationship between equicharacteristic étale sheaves and coherent $\mathrm{O}_{Y}$-modules. We will explain briefly the central ideas in the proof and then state the main result.

Let $\mathcal{M}$ be a coherent $\mathrm{O}_{Y}$-module which has a Frobenius-linear endomorphism $\phi: \mathcal{M} \rightarrow \mathcal{M}$. Then the subsheaf $\mathcal{M}^{\phi} \subseteq \mathcal{M}$ is a constructible $\mathbb{F}_{p}$-étale sheaf on $Y$. There is an exact sequence

$$
0 \longrightarrow M^{\phi} \longrightarrow M \stackrel{1-\phi}{\longrightarrow} M \longrightarrow 0 .
$$

This exact sequence determines a long exact sequence of cohomology groups. The long exact sequence can be used to prove

$$
\chi\left(Y, M^{\phi}\right) \geq \chi(Y, M) .
$$

This allows us to use results from coherent cohomology to study the Euler characteristic $\chi\left(Y, M^{\phi}\right)$. (This is an idea that is used in [Pink 2000].)

So, a general lower bound on $\chi(Y, N)$ is possible if there exists a canonical way to construct an exact sequence in the form of (1.0.3) for $N$. This paper offers such a canonical construction. The construction is based on the characteristic- $p$ "Riemann-Hilbert" correspondence of M. Emerton and M. Kisin [2004].

Let

$$
\mathcal{N}=\operatorname{Hom}_{\mathbb{F}_{p^{r}}}\left(N, \mathrm{O}_{Y}\right) .
$$

This sheaf is a quasicoherent $\mathrm{O}_{Y}$-module. Also, the $p$ th-power map on $\mathscr{O}_{Y}$ induces a Frobenius-linear endomorphism $\mathcal{N} \rightarrow \mathcal{N}$. In the notation of [Emerton and Kisin 2004], $\mathcal{N}$ is a left $\mathbb{O}_{F, Y}$-module. The sheaf $N$ can be recovered (except for sections with punctual support) from the sheaf $\mathcal{N}$. (See Theorem 2.3.11 in this paper.)

The sheaf $\mathcal{N}$ possesses special submodules which are called roots. A root of $\mathcal{N}$ is a coherent $O_{Y}$-submodule $\mathcal{N}^{\prime} \subseteq \mathcal{N}$ which satisfies some special properties (see Definition 2.1.3). The concept of a root is due to G. Lyubeznik [1997]. The special 
properties of a root imply in particular the existence of an exact sequence

$$
0 \longrightarrow N \longrightarrow\left(\mathcal{N}^{\prime}\right)^{\vee} \longrightarrow\left(\mathcal{N}^{\prime}\right)^{\vee} \longrightarrow 0,
$$

where $\left(\mathcal{N}^{\prime}\right)^{\vee}$ denotes the coherent sheaf dual of $\mathcal{N}^{\prime}$. Thus the Euler characteristic of $N$ is related to the Euler characteristics of the roots of $\mathcal{N}$. In this paper we show that the sheaf $\mathcal{N}$ possesses a canonical minimal root, $\mathcal{N}_{0}$, and that the Euler characteristic of $\mathcal{N}_{0}$ can be computed from local information about the sheaf $N{ }^{1}$ Inequality (1.0.4) thus gives a lower bound on $\chi(Y, N)$. The following theorem is the result:

Theorem 1.0.7. Let $N$ be a constructible $\mathbb{F}_{p}$-étale sheaf on $Y$ whose sections all have open support. Then

$$
\chi(Y, N) \geq(1-g)(\operatorname{rank} N)-\sum_{y \in Y} \mathfrak{C}\left(N_{(y)}\right) .
$$

(See Corollary 3.1.18.) In (1.0.8), the expression $\mathfrak{C}\left(N_{(y)}\right)$ denotes a local invariant which is called the minimal root index of $N$ at $y$.

In this paper we present a proof of the above theorem using the characteristic- $p$ Riemann-Hilbert correspondence (a functorial relationship between $O_{F, Y}$-modules and $\mathbb{F}_{p}$-étale sheaves). This correspondence is developed in full generality in [Emerton and Kisin 2004]. Since we prefer to avoid the language of derived categories, we will not make direct use of the results from that paper. Instead we prove a miniature version of the correspondence which applies to curves. Our version includes both a local and global version of the Riemann-Hilbert correspondence, as well as functors relating the two. (See Theorems 2.3.11, 2.3.16, and 4.3.1.)

The body of the paper is divided into three sections. Section 2 develops basic theory for the study of $O_{F, X}$-modules and gives the statement of the characteristic$p$ Riemann-Hilbert correspondence. Section 3 proves the main result and offers some examples. It is shown in Section 3.3 that the main result is compatible with the previous results of Pink [2000]. Finally, Section 4 gives the full proof of the Riemann-Hilbert correspondence in dimension one.

1.1. Notation and conventions. Throughout this paper, let $p$ denote a prime and let $r$ denote a positive integer. Let $k$ be an algebraically closed field of characteristic $p$. Let $\mathbb{F}_{p^{r}}$ denote a finite field of $p^{r}$ elements. Fix an embedding $\mathbb{F}_{p^{r}} \hookrightarrow k$.

All sheaves are assumed to be sheaves on an étale site. Thus, if $X$ is a $k$-scheme, then $O_{X}$ denotes the étale structure sheaf of $X$. If $x$ is a $k$-point of $X$, then $\mathscr{O}_{X, x}$ denotes the étale stalk of $O_{X}$ at $x$.

\footnotetext{
${ }^{1}$ We note that the existence of canonical minimal roots was recently proved, independently, in a much broader context. See [Blickle 2008].
} 
If $X$ is a $k$-scheme, $x$ is a closed point of $X$, and $Q$ is an étale sheaf on $X$, then $Q_{(x)}$ denotes the pullback of $Q$ via the morphism

$$
\operatorname{Spec} \mathrm{O}_{X, x} \rightarrow X \text {. }
$$

If $X$ is a $k$-scheme and $\mathscr{R}$ is a sheaf of rings on $X$, then $\operatorname{Mod}(X, \mathscr{R})$ denotes the category of sheaves of left $\mathscr{R}$-modules on $X$.

If $S$ is a $k$-algebra, let $F_{S}: S \rightarrow S$ denote the Frobenius map. If $X$ is a $k$-scheme, let $F_{X}: X \rightarrow X$ denote the Frobenius endomorphism.

All schemes are assumed to be Noetherian and separated.

\section{Unit $\mathfrak{O}_{F^{r}, X}$-modules}

This section covers some preliminaries. The central objects of concern are quasicoherent sheaves that have Frobenius-linear endomorphisms. Following [Emerton and Kisin 2004], we consider these sheaves as modules over a particular sheaf of noncommutative rings (namely, $\mathscr{O}_{F^{r}, X}$ ).

2.1. Definitions. Let $S$ be a $k$-algebra. Then $S\left[F^{r}\right]$ denotes the twisted polynomial algebra over $S$ determined by the $r$ th Frobenius endomorphism $F_{S}^{r}: S \rightarrow S$. Elements of $S\left[F^{r}\right]$ are thus finite sums of the form

$$
\sum_{i \geq 0} s_{i} F^{r i}
$$

with $s_{i} \in S$, and multiplication is expressed by the rule $F^{r} s=s^{p^{r}} F^{r}$.

Likewise, let $Z$ denote a $k$-scheme. Then $\widehat{O}_{F^{r}, Z}$ denotes the sheaf of twisted polynomial rings determined by the $r$ th Frobenius endomorphism of $\mathrm{O}_{Z}$. If $U \subseteq Z$ is any open subset, then $\mathscr{O}_{F^{r}, Z}(U) \cong \mathscr{O}_{Z}(U)\left[F^{r}\right]$.

A left $\mathcal{O}_{F^{r}}, Z$-module is simply an $\mathfrak{O}_{Z}$-module $\mathcal{N}$ equipped with an endomorphism $\phi: \mathcal{N} \rightarrow \mathcal{N}$ satisfying the condition

$$
\phi(f n)=f^{p^{r}} \phi(n) \quad \text { for any } U \subseteq Z, f \in \mathscr{O}_{Z}(U), n \in \mathcal{N}(U) .
$$

(The map $\phi$ is determined by the left-action of $F^{r}$.) This condition can be compactly expressed by saying that $\phi$ induces an $\mathrm{O}_{Z}$-linear morphism $F_{Z}^{r *} \mathcal{N} \rightarrow \mathcal{N}$.

Now we introduce some terminology from [Emerton and Kisin 2004]. If $\mathcal{N}$ is

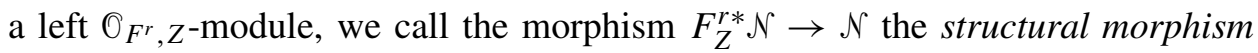

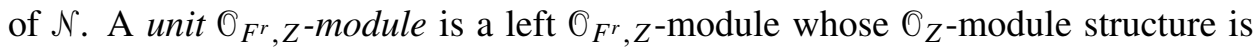
quasicoherent and whose structural morphism is an isomorphism. A unit $\mathbb{O}_{F^{r}}, Z^{-}$

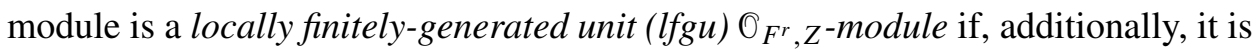
finitely-generated as a left $\mathrm{O}_{F^{r}}, Z$-module on any affine open subset of $Z$.

Let $\operatorname{Mod}^{u}\left(Z, \mathscr{O}_{F^{r}, Z}\right)$ and $\operatorname{Mod}^{f u}\left(Z, \mathscr{O}_{F^{r}, Z}\right)$ denote, respectively, the full sub-

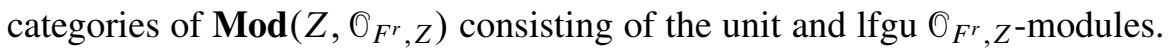


An lfgu $\mathscr{O}_{F^{r}}, Z$-module is not necessarily coherent, but it must have a coherent $\mathrm{O}_{Z}$-submodule which generates it under the action of $F^{r}$. The following definition identifies a special class of coherent generators for an lfgu $O_{F}{ }^{r} Z$-module. The definition is due to Lyubeznik.

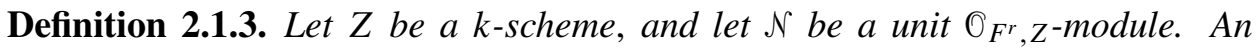
$\mathrm{O}_{Z}$-submodule $\mathcal{N}^{\prime} \subseteq \mathcal{N}$ is a root if

(1) the $\mathbb{O}_{Z}$-module $\mathcal{N}^{\prime}$ is coherent,

(2) the $\mathfrak{O}_{Z}$-submodule of $\mathcal{N}$ generated by $F^{r}\left(\mathcal{N}^{\prime}\right)$ contains $\mathcal{N}^{\prime}$, and

(3) as a left $\mathrm{O}_{F^{r}}, Z$-module, $\mathcal{N}$ is generated by $\mathcal{N}^{\prime}$.

Proposition 2.1.4. Let $W$ be a smooth irreducible $k$-scheme, and let $\mathscr{P}$ be an lfgu

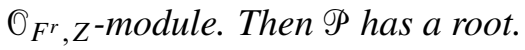

Proof. See [Emerton and Kisin 2004, Theorem 6.1.3].

Suppose that $W$ and $\mathscr{P}$ are as in the above proposition. Then the existence of a root for $\mathscr{P}$ allows us to express $\mathscr{P}$ as a union of coherent subsheaves. Let $\mathscr{P}_{0}$ be a root for $\mathscr{P}$, and let $\mathscr{P}_{i}$ denote the $\mathscr{O}_{Z}$-submodule of $\mathscr{P}$ generated by $F^{r i}\left(\mathscr{P}_{0}\right)$. The root properties imply that the subsheaves $\mathscr{P}_{i}$ form an ascending sequence

$$
\mathscr{P}_{0} \subseteq \mathscr{P}_{1} \subseteq \mathscr{P}_{2} \subseteq \cdots
$$

and $\bigcup \mathscr{P}_{i}=\mathscr{P}$.

Also, since we have assumed that $W$ is smooth, some stronger statements can be made. The flatness of the Frobenius morphisms $F_{W}^{r i}: W \rightarrow W$ implies that there are injections $F_{W}^{r i * \mathscr{P}_{0}} \rightarrow F_{W}^{r i * \mathscr{P}}$. Restricting the structural isomorphisms $F_{W}^{r i * \mathscr{P}} \rightarrow \mathscr{P}$ via these injections yields isomorphisms $F_{W}^{r i * \mathscr{P}_{0}} \rightarrow \mathscr{P}_{i}$. These isomorphisms imply that all terms in the sequence (2.1.5) have the same generic rank. In particular, since $\mathscr{P}_{0}$ and $\mathscr{P}_{1}$ have the same generic rank, they concur on some dense open subset $U \subseteq W$. The filtration (2.1.5) therefore collapses:

$$
\mathscr{P}_{0 \mid U}=\mathscr{P}_{1 \mid U}=\mathscr{P}_{2 \mid U}=\cdots .
$$

We have proved the following useful proposition.

Proposition 2.1.7. Let $W$ be a smooth irreducible $k$-scheme, and let $\mathscr{P}$ be an lfgu $\bigcirc_{F^{r}, W}$-module. There must exist a dense open subset $U \subseteq W$ on which $\mathscr{P}$ is coherent.

The same reasoning applied to one-point schemes also proves the following:

Proposition 2.1.8. Let $L$ be a field of characteristic $p$, and let $V$ be a finitelygenerated unit $L\left[F^{r}\right]$-module. Then $V$ is finite-dimensional over $L$. 
2.2. The minimal root. We will focus now on one-dimensional schemes. Let $Y$ denote a smooth projective $k$-curve.

Proposition 2.2.1. Let $\mathcal{N}$ be an lfgu $\mathcal{O}_{F^{r}, Y}$-module. Let $\mathcal{N}_{0}$ be a root for $\mathcal{N}$ which has minimal degree (as a coherent sheaf). Then $\mathcal{N}_{0}$ is contained in every other root of $\mathcal{N}$.

Proof. Suppose that $\mathcal{N}_{0}^{\prime}$ is another root for $\mathcal{N}$. Let $\mathcal{N}_{0}^{\prime \prime}=\mathcal{N}_{0}^{\prime} \cap \mathcal{N}_{0}$. I claim that $\mathcal{N}_{0}^{\prime \prime}$ is also a root for $\mathcal{N}$. This can be seen as follows: let $\left\{\mathcal{N}_{i}\right\}_{i=0}^{\infty},\left\{\mathcal{N}_{i}^{\prime}\right\}_{i=0}^{\infty}$, and $\left\{\mathcal{N}_{i}^{\prime \prime}\right\}_{i=0}^{\infty}$ denote the induced filtrations for $\mathcal{N}$ (described in Section 2.1). For any $i$, the module $\mathcal{N}_{i}^{\prime \prime}$ is the image under the isomorphism

$$
F_{Y}^{r i *} \mathcal{N} \rightarrow \mathcal{N}
$$

of $F_{Y}^{r i *}\left(\mathcal{N}_{0} \cap \mathcal{N}_{0}^{\prime}\right)$. By the flatness of the Frobenius morphism,

$$
F_{Y}^{r i *}\left(\mathcal{N}_{0} \cap \mathcal{N}_{0}^{\prime}\right)=\left(F_{Y}^{r i *} \mathcal{N}_{0}\right) \cap\left(F_{Y}^{r i *} \mathcal{N}_{0}^{\prime}\right)
$$

This equality implies that $\mathcal{N}_{i}^{\prime \prime}=\mathcal{N}_{i} \cap \mathcal{N}_{i}^{\prime}$. The root properties for $\mathcal{N}_{0}^{\prime \prime}$ follow immediately from this fact. Therefore $\mathcal{N}_{0}^{\prime \prime}$ is indeed a root. Since $\mathcal{N}_{0}^{\prime \prime}$ is contained in $\mathcal{N}_{0}$ and its degree cannot be any smaller than that of $\mathcal{N}_{0}$, we must have $\mathcal{N}_{0}=\mathcal{N}_{0}^{\prime \prime}$. This proves the proposition.

We will refer to the root of minimal degree simply as the "minimal root" of the lfgu $O_{F^{r}, Y}$-module. The minimal root also has a local description, which we now construct. Note that for any unit $\mathcal{O}_{F^{r}, Y}$-module $\mathcal{N}$, the stalk $\mathcal{N}_{y}$ at any closed point $y$ is a unit $\mathcal{O}_{Y, y}\left[F^{r}\right]$-module. If $\mathcal{N}_{0}$ is a root of $\mathcal{N}$, then $\left(\mathcal{N}_{0}\right)_{y}$ is a root of $\mathcal{N}_{y}$. We define an invariant for these local roots.

Definition 2.2.4. Let $(A, \mathfrak{m})$ be a Henselian $D V R$ of characteristic $p$ with $A / \mathfrak{m} \cong k$. Let $Q$ be a finitely-generated unit $A\left[F^{r}\right]$-module, and let $Q_{0} \subseteq Q$ be a root for $Q$. Let $Q_{1} \subseteq Q$ denote the A-submodule generated by $F^{r}\left(Q_{0}\right)$. Then the $F^{r}$-index of $Q_{0}$ is

$$
\frac{\operatorname{dim}_{k} Q_{1} / Q_{0}}{p^{r}-1}
$$

Proposition 2.2.6. Let $(A, \mathfrak{m})$ be a Henselian DVR of characteristic $p$ with $A / \mathfrak{m} \cong$ $k$. Let $Q$ be a finitely-generated unit $A\left[F^{r}\right]$-module. Then $Q$ has a unique minimal root which is contained in every other root.

Proof. This is a special case of [Blickle 2004, Theorem 2.10]. Alternatively, one can use Definition 2.2.4. Let $Q_{0} \subseteq Q$ be the root with the smallest $F^{r}$-index. Then $Q_{0}$ must be contained in every other root by an argument similar to the proof of Proposition 2.2.1. 
Definition 2.2.7. Let $A$ and $Q$ be defined as in Definition 2.2.4. Then the minimal root index of $Q$ (denoted $\mathfrak{C}(Q))$ is the $F^{r}$-index of the unique minimal root of $Q$.

We note the following basic proposition. The proof is elementary and is left to the reader.

Proposition 2.2.8. Let $(A, \mathfrak{m})$ be a Henselian $D V R$ with $A / \mathfrak{m} \cong k$, and let $Q$ be a unit $A\left[F^{r}\right]$-module which is finitely-generated over $A$. Then the only root of $Q$ is $Q$ itself. The minimal root index of $Q$ is 0 .

Using Proposition 2.2.6, we can characterize global minimal roots by their stalks:

Proposition 2.2.9. Let $\mathcal{N}$ be an lfgu $\mathcal{O}_{F^{r}, Y}$-module, and let $\mathcal{N}_{0}$ be the minimal root for $\mathcal{N}$. Then for any closed point $y \in Y$, the stalk $\left(\mathcal{N}_{0}\right)_{y}$ is the unique minimal root of $\mathcal{N}_{y}$.

Proof. This follows easily using Proposition 2.1.7. Let $V \subseteq Y$ be a nonempty open subcurve on which $\mathcal{N}$ is coherent. Then a coherent subsheaf $\mathcal{N}_{0}^{\prime} \subseteq \mathcal{N}$ is a root if and only if both of the following properties hold:

(1) $\left(\mathcal{N}_{0}^{\prime}\right)_{\mid V}=\mathcal{N}_{\mid V}$, and

(2) $\left(\mathcal{N}_{0}^{\prime}\right)_{y}$ is a root of $\mathcal{N}_{y}$ for every $y \in Y \backslash V$.

The minimal root $\mathcal{N}_{0}$ is simply the root which has a minimal stalk at every point of $Y \backslash V$.

The compatibility of local and global minimal roots implies an important numerical relationship. Let $\mathcal{N}$ be an lfgu $\mathscr{O}_{F^{r}, Y}$-module and $\mathcal{N}_{0} \subseteq \mathcal{N}$ be its minimal root. Let $\mathcal{N}_{1} \subseteq \mathcal{N}$ denote the $\mathcal{O}_{Y}$-submodule generated by $F^{r}\left(\mathcal{N}_{0}\right)$. Then $\mathcal{N}_{0} / \mathcal{N}_{1}$ is a skyscraper sheaf whose stalk-dimension at any point $y$ is equal to $\left(p^{r}-1\right) \mathfrak{C}\left(\mathcal{N}_{y}\right)$. Therefore

$$
\operatorname{deg} \mathcal{N}_{1}=\operatorname{deg} \mathcal{N}_{0}+\left(p^{r}-1\right) \sum_{y \in Y} \mathfrak{C}\left(\mathcal{N}_{y}\right) .
$$

On the other hand, the isomorphism $F_{Y}^{r *} \mathcal{N}_{0} \cong \mathcal{N}_{1}$ implies

$$
\operatorname{deg} \mathcal{N}_{1}=p^{r} \cdot \operatorname{deg} \mathcal{N}_{0} .
$$

Combining these two equalities yields

$$
\operatorname{deg} \mathcal{N}_{0}=\sum_{y \in Y} \mathfrak{C}\left(\mathcal{N}_{y}\right) .
$$

This formula will be important in Section 3. 
2.3. Unit $\mathrm{O}_{F, X}$-modules and étale sheaves. We continue to let $Y$ denote a smooth projective $k$-curve. Let $\mathcal{N}$ be an lfgu $\mathcal{O}_{F^{r}, Y}$-module. Then the sheaf

$$
\operatorname{Hom}_{\mathbb{O}_{F}, Y}\left(\mathcal{N}, O_{Y}\right)
$$

has the structure of an étale sheaf of $\mathbb{F}_{p^{r}}$-vector spaces on $Y$. Thus there is a functor

$$
\operatorname{Hom}_{\mathfrak{O}_{F^{r}, Y}}\left(\cdot, \mathfrak{O}_{Y}\right): \operatorname{Mod}^{f u}\left(Y, \mathscr{O}_{F^{r}, Y}\right) \rightarrow \operatorname{Mod}\left(Y, \mathbb{F}_{p^{r}}\right) .
$$

This functor has an alternative expression in terms of roots. Let $\mathcal{N}_{0}$ be a root for $\mathcal{N}$. Let $\mathcal{N}_{0}^{\vee}$ be the coherent sheaf dual for $\mathcal{N}_{0}$. There is a natural left $\mathcal{O}_{F^{r}, Y}$-module structure on the sheaf $\mathcal{N}_{0}^{\vee}$, which can be described as follows. Let $\mathcal{N}_{0} \subseteq \mathcal{N}_{1} \subseteq \mathcal{N}_{2} \subseteq$ $\cdots \subseteq \mathcal{N}$ denote the filtration induced by $\mathcal{N}_{0}$. For any homomorphism $\phi: \mathcal{N}_{0} \rightarrow \mathcal{O}_{Y}$, let $F^{r}(\phi) \in \mathcal{N}_{0}^{\vee}$ be the composition

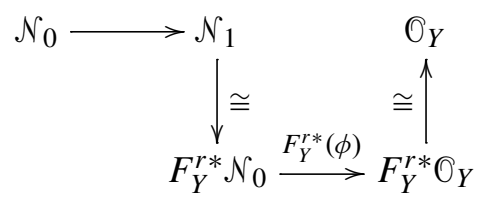

This assignment makes $\mathcal{N}_{0}^{\vee}$ a coherent (not necessarily unit) left $\mathcal{O}_{F^{r}, Y}$-module.

Consider the restriction map

$$
\operatorname{Hom}_{\mathscr{O}_{F}, Y}\left(\mathcal{N}, \mathscr{O}_{Y}\right) \rightarrow \operatorname{Hom}_{\mathscr{O}_{Y}}\left(\mathcal{N}_{0}, \mathscr{O}_{Y}\right)\left(=\mathcal{N}_{0}^{\vee}\right)
$$

Sections in the image of this map arise from Frobenius invariant morphisms from $\mathcal{N}$ to $O_{Y}$, and are therefore invariant under the action of $F^{r}$ on $\mathcal{N}_{0}^{V}$. Thus restriction actually determines a map

$$
\operatorname{Hom}_{\mathscr{O}_{F}, Y}\left(\mathcal{N}, \mathscr{O}_{Y}\right) \rightarrow\left(\mathcal{N}_{0}^{\vee}\right)^{F^{r}}
$$

On the other hand, suppose that $\psi: \mathcal{N}_{0} \rightarrow \mathcal{O}_{Y}$ is a homomorphism which is invariant under $F^{r}$. Then $\psi$ determines a series of maps

$$
\mathcal{N}_{i} \longrightarrow F_{Y}^{r i *} \mathcal{N}_{0} \stackrel{F_{Y}^{r i *}(\psi)}{\longrightarrow} \mathrm{O}_{Y}, i=0,1,2, \ldots
$$

which are all compatible. Taken together these determine a homomorphism $\mathcal{N} \rightarrow$ $\mathrm{O}_{Y}$ which is $\hat{O}_{F^{r}, Y}$-linear. This association is an inverse to (2.3.5), and thus we see that there is in fact an isomorphism,

$$
\operatorname{Hom}_{\mathbb{F}_{F}, Y}\left(\mathcal{N}, \mathscr{O}_{Y}\right) \cong\left(\mathcal{N}_{0}^{\vee}\right)^{F^{r}} .
$$

So, the sheaf $\operatorname{Hom}_{\mathscr{O}_{F}, Y}\left(\mathcal{N}, \mathscr{O}_{Y}\right)$ can be identified with a subsheaf of the coherent $\mathrm{O}_{X}$-module $\mathcal{N}_{0}^{\vee}$. 
The relationship between étale sheaves of $\mathbb{E}_{p^{r}}$-vector spaces and lfgu $\mathbb{O}_{F^{r}, Y^{-}}$ modules can be more fully understood with the addition of a second functor. Let

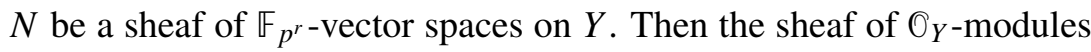

$$
\operatorname{Hom}_{\mathbb{F}_{p^{r}}}\left(N, \mathrm{O}_{Y}\right)
$$

has a natural structure of a left $\widehat{O}_{F^{r}, Y}$-module given by the Frobenius endomorphism of $\mathrm{O}_{Y}$. Thus there is a functor

$$
\operatorname{Hom}_{\mathbb{F}_{p^{r}}}\left(\cdot, \mathscr{O}_{Y}\right): \operatorname{Mod}\left(Y, \mathbb{F}_{p^{r}}\right) \rightarrow \operatorname{Mod}\left(Y, \mathscr{O}_{F^{r}, Y}\right) .
$$

If $\mathcal{N}$ is the lfgu $\mathscr{O}_{F^{r}, Y}$-module from above, then the sheaf

$$
\mathcal{N}^{\prime \prime}:=\operatorname{Hom}_{\mathbb{F}_{p^{r}}}\left(\operatorname{Hom}_{\mathbb{O}_{F^{r}, Y}}\left(\mathcal{N}, O_{Y}\right), O_{Y}\right)
$$

is a left $\widehat{O}_{F^{r}, Y}$-module. There is also a natural "double-dual" homomorphism $\mathcal{N} \rightarrow \mathcal{N}^{\prime \prime}$. The functors $\operatorname{Hom}_{\mathbb{C}_{F^{r}, Y}}\left(\cdot, \mathscr{O}_{Y}\right)$ and $\operatorname{Hom}_{\mathbb{F}_{p^{r}}}\left(\cdot, \mathscr{O}_{Y}\right)$ are the basis for the characteristic- $p$ Riemann-Hilbert correspondence.

We quote here (for use in Section 3) three results which summarize the RiemannHilbert correspondence on a curve. The first two results are proved in Section $4 .^{2}$ The third result follows from the first via isomorphism (2.3.7).

The Riemann-Hilbert correspondence on $Y$ is an equivalence between two categories: the category of torsion-free 1 fgu $O_{F^{r}, Y}$-modules, and the category of constructible $\mathbb{F}_{p^{r}}$-sheaves on $Y$ in which all sections have open support.

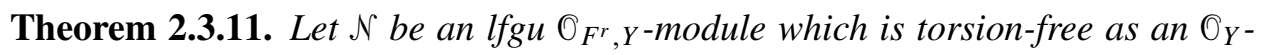
module. Then the sheaf

$$
\operatorname{Hom}_{\mathbb{O}_{F}, Y}\left(\mathcal{N}, \mathrm{O}_{Y}\right)
$$

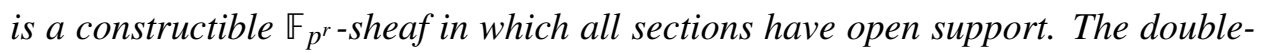
dual homomorphism

$$
\mathcal{N} \rightarrow \operatorname{Hom}_{\mathbb{F}_{p^{r}}}\left(\mathcal{H} \operatorname{mom}_{\mathfrak{O}_{F}, Y}\left(\mathcal{N}, \mathscr{O}_{Y}\right), \mathscr{O}_{Y}\right)
$$

is an isomorphism.

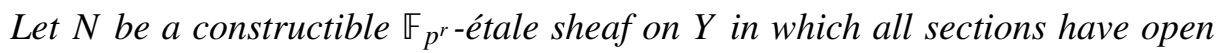
support. Then the sheaf

$$
\operatorname{Hom}_{\mathbb{F}_{p^{r}}}\left(N, \mathrm{O}_{Y}\right)
$$

is an lfgu $\mathfrak{O}_{F^{r}, Y}$-module which has no $\mathfrak{O}_{Y}$-torsion. The double-dual homomorphism

$$
N \rightarrow \operatorname{Hom}_{\mathfrak{O}_{F} r, Y}\left(\operatorname{Hom}_{\mathbb{F}_{p} r}\left(N, O_{Y}\right), \mathscr{O}_{Y}\right)
$$

is an isomorphism.

${ }^{2}$ Theorem 2.3.11 is proved in Section 4.5. Theorem 2.3.16 is a combination of Propositions 4.4.1 and 4.4.6. 


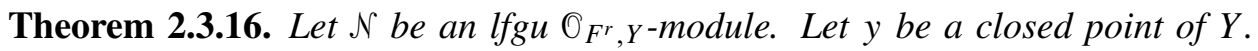
Then the natural homomorphism

$$
\operatorname{Hom}_{\mathscr{O}_{F}, Y}\left(\mathcal{N}, O_{Y}\right)_{y} \rightarrow \operatorname{Hom}_{\mathscr{O}_{Y, y}}\left[F^{r}\right]\left(\mathcal{N}_{y}, \mathscr{O}_{Y, y}\right)
$$

is an isomorphism.

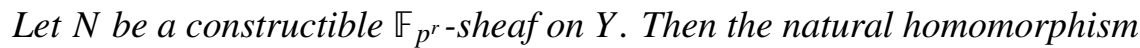

$$
\operatorname{Hom}_{\mathbb{F}_{p^{r}}}\left(N, \mathrm{O}_{Y}\right)_{y} \rightarrow \operatorname{Hom}_{\mathbb{F}_{p^{r}}}\left(N_{(y)}, \mathcal{O}_{\mathrm{Spec}} \mathrm{O}_{Y, y}\right)
$$

is an isomorphism. ${ }^{3}$

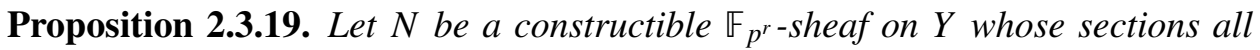
have open support. Let

$$
\mathcal{N}=\operatorname{Hom}_{\mathbb{F}_{p^{r}}}\left(N, \mathrm{O}_{Y}\right) \text {, }
$$

and let $\mathcal{N}_{0}$ be a root for $\mathcal{N}$. Then there is an isomorphism,

$$
N \stackrel{\cong}{\longrightarrow}\left(\mathcal{N}_{0}^{\vee}\right)^{F^{r}}
$$

\section{The Euler characteristic of an étale $\mathbb{F}_{p^{r}}$-sheaf}

Throughout this section, let $Y$ denote a smooth projective $k$-curve.

If $N$ is a constructible $\mathbb{F}_{p^{r}}$-sheaf on $Y$, let

$$
\chi(Y, N)=\operatorname{dim}_{\mathbb{F}_{p^{r}}} H^{0}(Y, N)-\operatorname{dim}_{\mathbb{F}_{p^{r}}} H^{1}(Y, N) .
$$

This section establishes a lower bound on $\chi(Y, N)$ using the theory developed in Section 2.

3.1. Main result. The following lemma provides the final preparation for the main theorem.

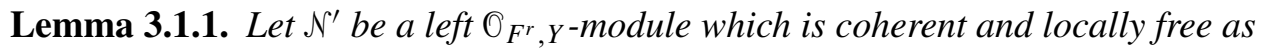
an $\mathrm{O}_{Y}$-module. Then the morphism

$$
\left(1-F^{r}\right)(\cdot): \mathcal{N}^{\prime} \rightarrow \mathcal{N}^{\prime}
$$

is surjective.

Proof. Let $y$ be a closed point of $Y$. We will show that morphism (3.1.2) is surjective at $y$. Choose any étale neighborhood $U \rightarrow Y$ of $y$, and any section $n \in \mathcal{N}^{\prime}(U)$. By replacing $U$ with a subordinate neighborhood if necessary, we may assume that $\mathcal{N}^{\prime}$ is globally free on $U$. We may also assume that $U$ is affine. Let $U=\operatorname{Spec} S$.

\footnotetext{
${ }^{3}$ Here $N_{(y)}$ denotes the pullback of $N$ via the natural morphism Spec ${ }^{O} Y, y \rightarrow Y$.
} 
Let $\left\{e_{1}, \ldots, e_{\ell}\right\}$ be a basis for the free $S$-module $\mathcal{N}^{\prime}(U)$. Choose elements $\left\{s_{i j} \mid\right.$ $1 \leq i \leq \ell, 1 \leq j \leq \ell\}$ such that

$$
F^{r}\left(e_{i}\right)=\sum_{j} s_{i j} e_{j}
$$

Let $n=\sum_{k=1}^{\ell} a_{k} e_{k}$, with $a_{k} \in S$.

Let $V \rightarrow U$ be the morphism of affine schemes defined by the ring extension

$$
S^{\prime}:=S\left[T_{1}, T_{2}, \ldots, T_{\ell}\right] /\left\langle\left\{T_{j}-a_{j}-\sum_{i} T_{i}^{p^{r}} s_{i j}\right\}_{j=1}^{\ell}\right\rangle .
$$

Note that this is an étale extension (since the module of relative differentials vanishes). The section

$$
\sum_{k} T_{k} \otimes e_{k} \in \mathcal{N}^{\prime}(V)
$$

maps to $n_{\mid V}$ under (3.1.2) (as the reader may verify). Therefore $n_{\mid V}$ is contained in the image of (3.1.2). Since $V \rightarrow U$ is finite and therefore surjective, $n$ itself is contained in the image of (3.1.2). This completes the proof.

Now we are ready to prove the main result. The basic method of our proof is borrowed from [Pink 2000].

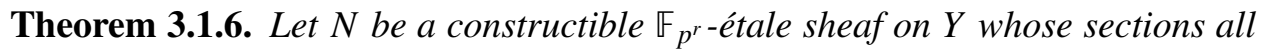
have open support. Let

$$
\mathcal{N}=\operatorname{Hom}_{\mathbb{F}_{p^{r}}}\left(N, \mathrm{O}_{Y}\right) .
$$

Let $g$ denote the genus $Y$, and let $m$ denote the rank of $N$. Then,

$$
\chi(Y, N) \geq(1-g) m-\sum_{y \in Y} \mathfrak{C}\left(\mathcal{N}_{y}\right) .
$$

Proof. Let $\mathcal{N}_{0}$ be the minimal root of $\mathcal{N}$, and let $\mathcal{N}_{0}^{\vee}$ denote its coherent dual. The sheaf $\mathcal{N}_{0}^{\vee}$ has the structure of a left $\mathcal{O}_{F^{r}, Y}$-module (as discussed in Section 2.3). By Proposition 2.3.19 and Lemma 3.1.1, this gives an exact sequence

$$
0 \longrightarrow N \longrightarrow \mathcal{N}_{0}^{\vee} \stackrel{1-F^{r}}{\longrightarrow} \mathcal{N}_{0}^{\vee} \longrightarrow 0
$$

Therefore the cohomology groups of $N$ and $\mathcal{N}_{0}^{\vee}$ are related by the long exact sequence

$$
\begin{aligned}
0 \longrightarrow & H^{0}(Y, N) \longrightarrow H^{0}\left(Y, \mathcal{N}_{0}^{\vee}\right) \longrightarrow H^{0}\left(Y, \mathcal{N}_{0}^{\vee}\right) \longrightarrow \\
& H^{1}(Y, N) \longrightarrow H^{1}\left(Y, \mathcal{N}_{0}^{\vee}\right) \longrightarrow H^{1}\left(Y, \mathcal{N}_{0}^{\vee}\right) \longrightarrow
\end{aligned}
$$


The finite-dimensional $k$-vector spaces $H^{0}\left(Y, \mathcal{N}_{0}^{\vee}\right)$ and $H^{1}\left(Y, \mathcal{N}_{0}^{\vee}\right)$ are left $k\left[F^{r}\right]$ modules, the first one being unit. There exist left $k\left[F^{r}\right]$-module isomorphisms

$$
\begin{aligned}
& H^{0}\left(Y, \mathcal{N}_{0}^{\vee}\right) \cong k^{\oplus t_{0}}, \\
& H^{1}\left(Y, \mathcal{N}_{0}^{\vee}\right) \cong k^{\oplus t_{1}} \oplus W,
\end{aligned}
$$

where $W$ is a left $k\left[F^{r}\right]$-module with a nilpotent $F^{r}$-action. (See [Deligne and Katz 1973, Section 1] for a discussion of this type of decomposition.)

Based on these isomorphisms, we can see that the map

$$
\left(1-F^{r}\right)(\cdot): H^{0}\left(Y, \mathcal{N}_{0}^{\vee}\right) \rightarrow H^{0}\left(Y, \mathcal{N}_{0}^{\vee}\right)
$$

is surjective, and therefore (3.1.10) splits up into two short exact sequences:

$$
\begin{aligned}
& 0 \longrightarrow H^{0}(Y, N) \longrightarrow H^{0}\left(Y, \mathcal{N}_{0}^{\vee}\right) \stackrel{1-F^{r}}{\longrightarrow} H^{0}\left(Y, \mathcal{N}_{0}^{\vee}\right) \longrightarrow 0 \\
& 0 \longrightarrow H^{1}(Y, N) \longrightarrow H^{1}\left(Y, \mathcal{N}_{0}^{\vee}\right) \stackrel{1-F^{r}}{\longrightarrow} H^{1}\left(Y, \mathcal{N}_{0}^{\vee}\right) \longrightarrow 0
\end{aligned}
$$

We can see further that the $\mathbb{F}_{p^{r}}$-dimension of $H^{0}(Y, N)$ is the same as the $k$ -

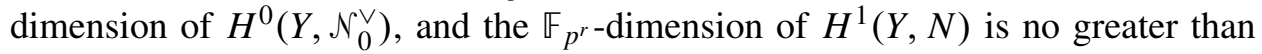
the $k$-dimension of $H^{1}\left(Y, \mathcal{N}_{0}^{\vee}\right)$. Thus we obtain the inequality

$$
\chi(Y, N) \geq \chi\left(Y, \mathcal{N}_{0}^{\vee}\right)
$$

where $\chi\left(Y, \mathcal{N}_{0}^{\vee}\right)=\operatorname{dim}_{k} H^{0}\left(Y, \mathcal{N}_{0}^{\vee}\right)-\operatorname{dim}_{k} H^{1}\left(Y, \mathcal{N}_{0}^{\vee}\right)$. Now Theorem 3.1.6 follows easily from the Riemann-Roch formula. By the discussion at the end of Section 2.2,

$$
\operatorname{deg} \mathcal{N}_{0}=\sum_{y \in Y} \mathfrak{C}\left(\mathcal{N}_{y}\right)
$$

Therefore by the Riemann-Roch theorem,

$$
\begin{aligned}
\chi(Y, N) & \geq \chi\left(Y, \mathcal{N}_{0}^{\vee}\right)=(1-g) m+\operatorname{deg} \mathcal{N}_{0}^{\vee} \\
& =(1-g) m-\operatorname{deg} \mathcal{N}_{0}=(1-g) m-\sum_{y \in Y} \mathfrak{C}\left(\mathcal{N}_{y}\right) .
\end{aligned}
$$

An alternate (and perhaps more interesting) formulation of Theorem 3.1.6 is allowed if we extend some of our notation. If $N$ is a constructible $\mathbb{F}_{p^{r}}$-sheaf on $Y$, and $y$ is a closed point of $Y$, then let $\mathfrak{C}\left(N_{(y)}\right)$ denote the minimal root index of the Riemann-Hilbert dual

$$
\operatorname{Hom}_{\mathbb{F}_{p^{r}}}\left(N_{(y)}, \mathscr{O}_{\mathrm{Spec}} \mathscr{O}_{Y, y}\right) .
$$

By Theorem 2.3.16, this dual is isomorphic to $\mathcal{N}_{y}$. The following corollary follows immediately. 


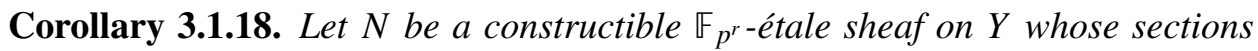
all have open support. Let $g$ denote the genus of $Y$ and $m$ the rank of $N$. Then,

$$
\chi(Y, N) \geq(1-g) m-\sum_{y \in Y} \mathfrak{C}\left(N_{(y)}\right) .
$$

3.2. Examples. We will illustrate Theorem 3.1 .6 and Corollary 3.1 .18 via two examples on the projective line.

We begin with a brief discussion in the local setting. As it turns out, the local terms from Corollary 3.1.18 are easy to calculate explicitly in the case of rank-one sheaves. Let $y$ be a closed point of $Y$. Let $A=\mathcal{O}_{Y, y}$, and let $K$ be the fraction field of $A$. Let $t \in K$ be a local parameter. Let $K^{\prime} / K$ be a degree- $\left(p^{r}-1\right)$ extension having an element $t^{\prime}$ which satisfies $\left(t^{\prime}\right)^{p^{r}-1}=t$.

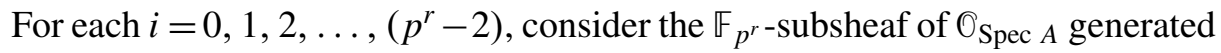
by the element $\left(t^{\prime}\right)^{i} \in K^{\prime}$. Call this sheaf $V_{i}$. Each such sheaf is a rank-one $\mathbb{F}_{p^{r}}$-sheaf with no global sections. The sheaves $V_{0}, V_{1}, \ldots, V_{p^{r}-2}$ taken together express all of the isomorphism types of nontrivial rank-one $\mathbb{F}_{p^{r}}$-sheaves on Spec $A$.

Now consider the set of all $\mathbb{F}_{p^{r}}$-linear morphisms from $V_{i}$ into $O_{\text {Spec } A}$. One such morphism is simply the inclusion $f_{i}: V_{i} \hookrightarrow O_{\text {Spec } A}$. The Riemann-Hilbert dual

$$
\mathscr{V}_{i}=\operatorname{Hom}_{\mathbb{F}_{p^{r}}}\left(V_{i}, \mathcal{O}_{\operatorname{Spec} A}\right)
$$

is a one-dimensional $K$-vector space generated by the morphism $f_{i}$. As the reader may verify, the left $A\left[F^{r}\right]$-module structure of $\mathscr{V}_{i}$ is given by

$$
F^{r}\left(f_{i}\right)=t^{i} \cdot f_{i}
$$

Calculating the minimal root index for $V_{i}$ is thus straightforward. The smallest $A$-module in $\mathscr{V}_{i}$ which satisfies the root properties is the $A$-module generated by $t^{-1} f_{i}$. The $F^{r}$-index of this module is $1-i /\left(p^{r}-1\right)$. Therefore,

$$
\mathfrak{C}\left(V_{i}\right)=1-\frac{i}{p^{r}-1}
$$

In the examples that follow, we will use the following notation: if $Z$ is a $k$ -

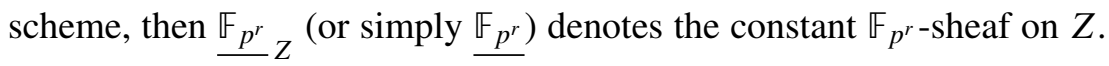

Example 3.2.4. Suppose that $p>2$. Let $\mathbb{P}^{1}$ denote the projective line over $k$. Let $\alpha: \mathbb{P}^{1} \rightarrow \mathbb{P}^{1}$ denote the double-cover of $\mathbb{P}^{1}$ which maps 0 to $0, \infty$ to $\infty$, and is ramified at both of those points. Define

$$
N=\alpha_{*}\left(\underline{\mathbb{F}_{p^{r}} \mathbb{P}^{1}}\right) .
$$


The sheaf $N$ is a rank-2 sheaf which is locally constant at every point except for 0 and $\infty$. The stalk $N_{\infty}$ decomposes as a direct sum

$$
N_{\infty} \cong W \oplus W^{\prime},
$$

in which $W$ is a trivial rank-one sheaf, and $W^{\prime}$ is isomorphic to the sheaf $V_{\left(p^{r}-1\right) / 2}$ from the classification above. Therefore,

$$
\mathfrak{C}\left(N_{\infty}\right)=\mathfrak{C}(W)+\mathfrak{C}\left(W^{\prime}\right)=\frac{1}{2}
$$

A similar calculation shows that $\mathfrak{C}\left(N_{0}\right)=\frac{1}{2}$.

In this case, Corollary 3.1.18 asserts:

$$
\chi(Y, N) \geq(1-0) \cdot 2-\sum_{y \in Y} \mathfrak{C}\left(N_{(y)}\right)=2-\left(\frac{1}{2} \cdot 2\right)=1 .
$$

On the other hand, the Euler characteristic of $N$ can be calculated directly. The dimensions of the cohomology groups of $N=\alpha_{*} \underline{\mathbb{F}_{p^{r}}}$ are the same as those of $\underline{\mathbb{E}_{p^{r}}}$ (this can be seen from a Leray-Serre spectral sequence). Therefore $\chi\left(\mathbb{P}^{1}, N\right)=$ $\chi\left(\mathbb{P}^{1}, \mathbb{F}_{p^{r}}\right)=1$. So the formula from Corollary 3.1 .18 actually computes $\chi\left(\mathbb{P}^{1}, N\right)$ exactly.

Example 3.2.9. Now suppose $p>5$. Let $E$ be an elliptic curve, and let $\beta: E \rightarrow \mathbb{P}^{1}$ be a degree- 2 morphism which has 4 ramified points, each of ramification index 2 . Label the ramified points as $q_{1}, q_{2}, q_{3}, q_{4} \in \mathbb{P}^{1}$.

Let $N=\beta_{*}\left(\mathbb{F}_{p^{r}}\right)$. Then $N$ is locally constant on $\mathbb{P}^{1} \backslash\left\{q_{1}, q_{2}, q_{3}, q_{4}\right\}$, and by a calculation similar to the one in Example 3.2.4,

$$
\mathfrak{C}\left(N_{q_{i}}\right)=\frac{1}{2} \quad \text { for } i=1,2,3,4 .
$$

So Theorem 3.1.6 asserts

$$
\chi\left(\mathbb{P}^{1}, N\right) \geq(1-0) \cdot 2-\sum_{i=1}^{4} \mathfrak{C}\left(N_{q_{i}}\right)=0 .
$$

In actuality, $\chi\left(\mathbb{P}^{1}, N\right)$ is equal to $\chi\left(E, \mathbb{F}_{p^{r}}\right)$. This quantity can be equal to 0 or 1 , depending on whether on $E$ is a supersingular elliptic curve.

So, Corollary 3.1.18 yields an equality if and only if $E$ is an ordinary elliptic curve.

3.3. The minimal root index of a tame sheaf. The discussion at the beginning of the previous subsection will lead us to a more general calculation of the minimal root index. Again, let $y$ be a closed point of the curve $Y$, let $A=\mathrm{O}_{Y, y}$, and let $K$ be the fraction field of $A$. Let $t$ be a local parameter in $A$.

We need the following proposition and corollary. 
Proposition 3.3.1. Let $a$ and $b$ be positive integers. Let $Q$ be a finitely-generated unit $A\left[F^{a}\right]$-module. Then the minimal root index of $Q$ when considered as a unit $A\left[F^{a b}\right]$-module is the same as the minimal root index of $Q$ when considered as a unit $A\left[F^{a}\right]$-module.

Proof. Let $Q_{0} \subseteq\left({ }_{A\left[F^{a b}\right]} Q\right)$ be the minimal root for $Q$ considered as an $A\left[F^{a b}\right]$ module. For any $i \geq 0$, let $Q_{i / b}$ be the submodule of $Q$ generated by $F^{a i}\left(Q_{0}\right)$. The module $Q_{1 / b}$ is also a root for ${ }_{A\left[F^{a b}\right]} Q$. (This is clear via the isomorphism $F^{a *} Q \rightarrow$ $Q$.) Since both $Q_{0}$ and $Q_{1 / t}$ are roots for ${ }_{A\left[F^{a b}\right]} Q$, the intersection $Q_{0} \cap Q_{1 / b}$ is a root for ${ }_{A\left[F^{a b}\right]} Q$ (by similar reasoning to that in the proof of Proposition 2.2.1). Since $Q_{0}$ is the minimal root for ${ }_{A\left[F^{a b}\right]} Q$, it follows that $Q_{0} \cap Q_{1 / b}=Q_{0}$. Therefore $Q_{0} \subseteq Q_{1 / b}$, which means that $Q_{0}$ is a root not only for ${ }_{A\left[F^{a b}\right]} Q$ but for $Q$ itself.

The above reasoning shows that the minimal root for ${ }_{A\left[F^{a b}\right]} Q$ contains the minimal root for $Q$. But the reverse inclusion is obvious. So we see that $Q_{0}$ is the minimal root for both ${ }_{A\left[F^{a b}\right]} Q$ and $Q$. Thus,

$$
\begin{aligned}
\mathfrak{C}\left({ }_{A\left[F^{a b}\right]} Q\right) & =\frac{\operatorname{dim}_{k}\left(Q_{1} / Q_{0}\right)}{p^{a b}-1}=\frac{\sum_{i=0}^{b-1} \operatorname{dim}_{k}\left(Q_{(i+1) / b} / Q_{i / t}\right)}{p^{a b}-1} \\
& =\frac{\sum_{i=0}^{b-1}\left(p^{a i}\right) \operatorname{dim}_{k}\left(Q_{1 / b} / Q_{0}\right)}{p^{a b}-1}=\frac{\operatorname{dim}_{k}\left(Q_{1 / b} / Q_{0}\right)}{p^{a}-1}=\mathfrak{C}(Q) .
\end{aligned}
$$

Corollary 3.3.2. Let $W$ be a constructible $\mathbb{F}_{p^{a}-\text { sheaf on }}$ Spec $A$ whose sections all have open support. Then the minimal root index of the $\mathbb{F}_{p^{a b}-\text { sheaf }}$

$$
\mathbb{F}_{p^{a b}} \otimes_{\mathbb{F}_{p^{a}}} W
$$

is the same as the minimal root index of $W$.

Proof. Let $\mathcal{W}$ be the Riemann-Hilbert dual of $W$. Then the Riemann-Hilbert dual of $\mathbb{F}_{p^{a b}} \otimes W$ is ${ }_{A\left[F^{a b}\right]} W$.

Now, let $K^{\text {tame }} / K$ denote the direct limit of all tame extensions of $K$ (that is, extensions of order coprime to $p$ ). A sheaf on $\operatorname{Spec} A$ will be called a tame sheaf if its monodromy representation factors through $\operatorname{Gal}\left(K^{\mathrm{tame}} / K\right)$.

Every finite quotient of $\operatorname{Gal}\left(K^{\text {tame }} / K\right)$ is an abelian group of order coprime to $p$. Therefore, any finite-image representation of $\operatorname{Gal}\left(K^{\text {tame }} / K\right)$ over an algebraically closed field of characteristic $p$ decomposes into one-dimensional representations. This fact has a natural consequence for sheaves on $\operatorname{Spec} A$ : if $W$ is a tame con-

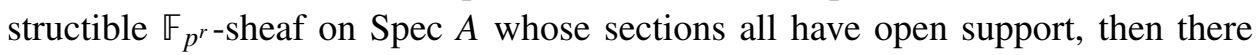
exists a field extension $\mathbb{F}_{p^{r b}} / \mathbb{F}_{p^{r}}$ such that the sheaf $\mathbb{F}_{p^{r b}} \otimes W$ decomposes into a

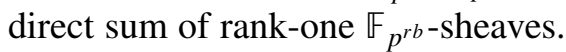

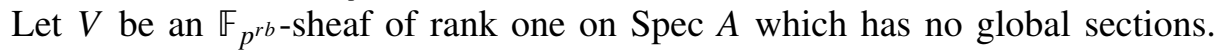
Then (as discussed in Section 3.2), there is a natural way to associate $V$ with a 


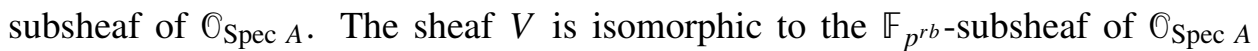
generated by some element $s \in K^{\text {tame }}$ which satisfies $s^{c}=t^{d}$ for some integers $c$ and $d$ with $0 \leq d<c, p \nmid c$.

Let $\lambda_{V}$ denote the quantity $\frac{d}{c}$. Then the minimal root index of $V$ is

$$
\mathfrak{C}(V)=1-\lambda_{V}
$$

The following proposition follows immediately.

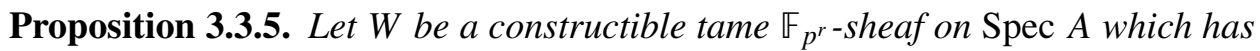
no global sections. Let $\mathbb{F}_{p^{r b}} / \mathbb{F}_{p^{r}}$ be a field extension such that $\mathbb{F}_{p^{r b}} \otimes W$ can be decomposed into a direct sum of rank-one sheaves. Then

$$
\mathfrak{C}(W)=\sum_{V}\left(1-\lambda_{V}\right)
$$

where the sum is taken over all summands in the decomposition of $\mathbb{F}_{p^{r b}} \otimes W$.

We note in passing that Proposition 3.3.5 makes the minimal root index compatible with the local terms used in [Pink 2000]. Specifically: if $N$ is a constructible $\mathbb{F}_{p^{r}}$-sheaf on $Y$ such that $N_{y}=\{0\}$ and $N_{(y)}$ is tame, the rational invariant $\operatorname{LT}_{y}^{\mathbb{F}_{p^{r}}} N$ [Pink 2000, Definition 5.3] is exactly equal to the minimal root index of $N_{(y)}$.

\section{The Riemann-Hilbert correspondence on a curve}

The purpose of this section is to prove the characteristic- $p$ Riemann-Hilbert correspondence in dimension one. Our approach is to build the proof in stages, beginning in the local setting and then proceeding to the global.

Throughout this section, let $Y$ denote a smooth irreducible projective $k$-curve.

4.1. The Riemann-Hilbert correspondence over a field. Suppose that $L_{0}$ is a separably closed field of characteristic $p$. Let $P_{0}$ be a finitely-generated unit $L_{0}\left[F^{r}\right]$ module. Then $P_{0}$ must have an $L_{0}$-basis which is invariant under the action of $F^{r}$. This basis determines an $L_{0}\left[F^{r}\right]$-module isomorphism $P_{0} \cong\left(L_{0}\right)^{\oplus d}$ for some $d \geq 0$ [Deligne and Katz 1973, Proposition 1.1].

More generally, if $L$ is any field of characteristic $p$, and $P$ is a finitely-generated unit $L\left[F^{r}\right]$-module, then there exists a finite separable extension $L^{\prime} / L$ such that $L^{\prime} \otimes_{L} P$ is a trivial $L^{\prime}\left[F^{r}\right]$-module.

The following proposition about sheaves on Spec $L$ follows easily.

Proposition 4.1.1. Let $L$ be a field of characteristic $p$, and let $T=\operatorname{Spec} L$. Then for any lfgu $\mathrm{O}_{F}{ }^{r}$, -module $\mathcal{V}$, the double-dual morphism

$$
\mathscr{V} \rightarrow \operatorname{Hom}_{\mathbb{F}_{p^{r}}}\left(\mathcal{H} m_{\mathbb{C}_{F}, T}\left(\mathcal{V}, O_{T}\right), \mathcal{O}_{T}\right)
$$




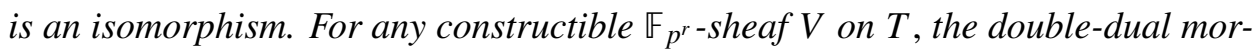
phism

$$
V \rightarrow \operatorname{Hom}_{\mathfrak{O}_{F}, T}\left(\operatorname{Hom}_{\mathbb{F}_{p^{r}}}\left(V, \mathrm{O}_{T}\right), \mathrm{O}_{T}\right)
$$

is an isomorphism.

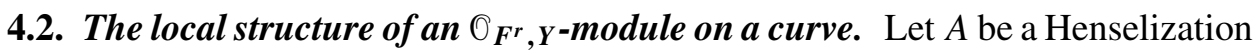
of the local ring $k[t]_{(t)}$. Let $K$ denote the fraction field of $A$. Note that for any closed point $y \in Y$, the local ring $O_{Y, y}$ is isomorphic to $A$. Therefore, we are interested in the structure of unit $A\left[F^{r}\right]$-modules.

We will refer to a unit $A\left[F^{r}\right]$-module as torsion-free if it has no $A$-torsion. Suppose that $Q$ is a torsion-free finitely-generated unit $A\left[F^{r}\right]$-module. Then let

$$
Q^{\mathrm{vec}}=\bigcap_{i=0}^{\infty} t^{i} Q .
$$

The module $Q^{\text {vec }}$ is the largest $K$-vector space contained in $Q$. This definition can be easily generalized to modules over any DVR. The reader may check the following elementary assertions:

(1) The module $Q^{\mathrm{vec}}$ is a finitely-generated unit $K\left[F^{r}\right]$-module.

(2) The quotient $Q / Q^{\mathrm{vec}}$ is a finitely-generated unit $A\left[F^{r}\right]$-module.

(3) For any finite integral extension of rings $A \hookrightarrow A^{\prime}$,

$$
\left(A^{\prime} \otimes_{A} Q\right)^{\mathrm{vec}}=A^{\prime} \otimes_{A} Q^{\mathrm{vec}} .
$$

There is an exact sequence

$$
0 \rightarrow Q^{\mathrm{vec}} \rightarrow Q \rightarrow Q / Q^{\mathrm{vec}} \rightarrow 0
$$

for any torsion-free finitely-generated unit $A\left[F^{r}\right]$-module $Q$. In this subsection we are going to show that the quotient $Q / Q^{\text {vec }}$ always has a trivial structure.

We begin with an algebraic lemma.

Lemma 4.2.4. Let $Q$ be a unit $A\left[F^{r}\right]$-module which is a free A-module of finite rank. Then, any $F^{r}$-invariant element of $Q / t Q$ can be uniquely lifted to an $F^{r}$ invariant element of $Q$.

Proof. The lemma may be formulated in terms of commutative algebra. Let $\left\{q_{1}, \ldots, q_{n}\right\}$ be any $A$-module basis for $Q$. Since $Q$ is a unit $A\left[F^{r}\right]$-module, the set $\left\{F^{r}\left(q_{1}\right), \ldots, F^{r}\left(q_{n}\right)\right\}$ is another basis, and there exists an invertible $A$-matrix $\left(c_{i j}\right)$ such that

$$
q_{i}=\sum_{j=1}^{n} c_{i j} F^{r}\left(q_{j}\right) .
$$


An element

$$
\sum_{k=1}^{n} a_{k} q_{k} \in Q, \quad\left(a_{k} \in A\right)
$$

is $F^{r}$-invariant if and only if

$$
\sum_{k=1}^{n} a_{k}^{p^{r}} F^{r}\left(q_{k}\right)=\sum_{k=1}^{n} a_{k} q_{k}=\sum_{k=1}^{n} a_{k} \sum_{j=1}^{n} c_{k j} F^{r}\left(q_{j}\right),
$$

or equivalently,

$$
a_{k}^{p^{r}}=\sum_{\ell=1}^{n} a_{\ell} c_{\ell k}
$$

for each $k=1,2, \ldots, n$. Let

$$
R=A\left[X_{1}, \ldots, X_{n}\right] /\left(\left\{X_{k}^{p^{r}}-\sum_{\ell=1}^{n} X_{\ell} c_{\ell k}\right\}_{k=1}^{n}\right) .
$$

Then $F^{r}$-invariant elements of $Q$ may be specified by $A$-homomorphisms from $R$ into $A$, while $F^{r}$-invariant elements of $Q / t Q$ may be specified by $A$-homomorphisms from $R$ into $k$. The claim made in the lemma, then, is equivalent to the assertion that every element of $\operatorname{Hom}_{A}(R, k)$ can be lifted to an element of $\operatorname{Hom}_{A}(R, A)$.

This assertion becomes evident once we understand the structure of $R$. The extension $A \rightarrow R$ is finite, flat, and unramified (as the reader may check), and therefore étale. Since $A$ is a Henselian local ring, $R$ is simply a finite direct sum of copies of $A$.

Proposition 4.2.10. Let $Q$ be a unit $A\left[F^{r}\right]$-module which is a free A-module of finite rank. Then there exists an $A\left[F^{r}\right]$-module isomorphism $Q \cong A^{\oplus d}$ for some d.

Proof. Choose an $F^{r}$-invariant $k$-basis for $Q / t Q$. There is a unique $F^{r}$-invariant lifting of this set to $Q$ by Lemma 4.2.4. By Nakayama's lemma, this lifting is an $A$-module basis, and so it determines the desired isomorphism.

Proposition 4.2.11. Suppose that $Q$ is a torsion-free unit $A\left[F^{r}\right]$-module for which there exists a $K\left[F^{r}\right]$-module isomorphism,

$$
K \otimes_{A} Q \cong K^{\oplus d}
$$

(with $d \geq 1$ ). Then there exists an $A\left[F^{r}\right]$-module isomorphism

$$
Q \cong K^{\oplus d^{\prime}} \oplus A^{\oplus\left(d-d^{\prime}\right)}
$$

for some $d^{\prime}$ with $0 \leq d^{\prime} \leq d$. 
Proof. Let $Q^{\prime} \subseteq K^{\oplus d}$ be the image of $Q$ under the injection $Q \rightarrow K \otimes_{A} Q \rightarrow K^{\oplus d}$. I claim first that $Q^{\prime} \supseteq\left(A^{\oplus d}\right)$. Suppose that this were not so. Then, the module

$$
S:=\left(A^{\oplus d}\right) /\left(Q^{\prime} \cap A^{\oplus d}\right)
$$

is a nontrivial unit $A\left[F^{r}\right]$-module. This module is a torsion $A$-module, and therefore has finite dimension over $k$. But then the structural isomorphism

$$
F_{A}^{r *} S \rightarrow S
$$

implies that $\left(p^{r}\right) \operatorname{dim}_{k} S=\operatorname{dim}_{k} S$, which is impossible. Therefore $Q^{\prime}$ contains $\left(A^{\oplus d}\right)$.

Now consider the intersection of $Q^{\prime}$ with the subset

$$
t^{-1}\left(k^{\oplus d}\right)=\left\{\left(t^{-1} c_{1}, \ldots, t^{-1} c_{d}\right) \mid c_{i} \in k\right\} \subseteq K^{\oplus d} .
$$

The intersection $Q^{\prime} \cap t^{-1}\left(k^{\oplus d}\right)$ has a Frobenius-linear endomorphism given by

$$
Q^{\prime} \cap t^{-1}\left(k^{\oplus d}\right) \stackrel{F^{r}(\cdot)}{\longrightarrow} Q^{\prime} \cap t^{-p^{r}}\left(k^{\oplus d}\right) \stackrel{\cdot\left(t^{p^{r}-1}\right)}{\longrightarrow} Q^{\prime} \cap t^{-1}\left(k^{\oplus d}\right) .
$$

We can find a $k$-basis $\left\{\lambda_{1}, \lambda_{2}, \ldots, \lambda_{d^{\prime}}\right\}$ for $Q^{\prime} \cap t^{-1}\left(k^{\oplus d}\right)$ consisting of elements which are fixed by this endomorphism. The elements in this basis can be written as

$$
\lambda_{i}=t^{-1} e_{i}
$$

where $e_{i} \in\left(\mathbb{F}_{p^{r}}\right)^{\oplus d} \subset K^{\oplus d}$.

Note that since $t^{-1} e_{i} \in Q^{\prime}$, the $A\left[F^{r}\right]$-module structure of $Q^{\prime}$ implies that $t^{-M} e_{i} \in Q^{\prime}$ for any $M>0$. Now enlarge the set $\left\{e_{1}, \ldots, e_{d^{\prime}}\right\}$ to a $d$-element set $\left\{e_{1}, \ldots, e_{d}\right\}$ which is a basis for $\left(\mathbb{F}_{p^{r}}\right)^{\oplus d}$. The basis $\left\{e_{1}, \ldots, e_{d}\right\}$ determines a map

$$
K^{\oplus d^{\prime}} \oplus A^{\oplus\left(d-d^{\prime}\right)} \rightarrow Q^{\prime},
$$

which is easily seen to be an isomorphism. This completes the proof.

Proposition 4.2.20. Let $Q$ be a torsion-free finitely-generated unit $A\left[F^{r}\right]$-module such that $Q^{\mathrm{vec}}=\{0\}$. Then there exists an $A\left[F^{r}\right]$-module isomorphism $Q \cong A^{\oplus d}$ for some $d \geq 0$.

Proof. The module $K \otimes_{A} Q$ is a finitely-generated unit $K\left[F^{r}\right]$-module. Choose a finite separable extension $K^{\prime} / K$ which trivializes $Q$. Let $A^{\prime} \subseteq K^{\prime}$ be the integral closure of $A$ in $K^{\prime}$. Let $Q^{\prime}=A^{\prime} \otimes_{A} Q$.

The module $Q^{\prime}$ is a torsion-free unit $A^{\prime}\left[F^{r}\right]$-module such that $Q^{\prime} \otimes_{A^{\prime}} K^{\prime}$ is isomorphic to $\left(K^{\prime}\right)^{\oplus d}$ for some $d$. Note that $A^{\prime}$, like $A$, is a Henselian local ring, 
and in fact there exists an isomorphism between the pair $\left(A^{\prime}, K^{\prime}\right)$ and $(A, K)$. Via this isomorphism, we can apply Proposition 4.2.11 to find an isomorphism

$$
Q^{\prime} \cong K^{\prime \oplus d^{\prime}} \oplus A^{\prime \oplus\left(d-d^{\prime}\right)} .
$$

Now since $Q^{\prime \text { vec }}=\{0\}$, clearly we must have $d^{\prime}=0$. Thus $Q^{\prime} \cong A^{\prime \oplus d}$.

The inclusion $Q \hookrightarrow Q^{\prime}$ makes $Q$ isomorphic to a submodule of a finitelygenerated $A$-module. Therefore $Q$ is itself a finitely-generated $A$-module. The desired result now follows from Proposition 4.2.10.

Proposition 4.2.22. Let $Q$ be a torsion-free finitely-generated unit $A\left[F^{r}\right]$-module. Then $Q / Q^{\mathrm{vec}}$ is isomorphic to $A^{\oplus d}$ for some $d \geq 0$.

Proof. Immediate from Proposition 4.2.20.

4.3. The local Riemann-Hilbert correspondence. We now prove the RiemannHilbert correspondence over the local rings $\mathrm{O}_{Y, y}$.

Theorem 4.3.1. Let $y$ be a closed point of $Y$, and let $Z=\operatorname{Spec}_{O_{Y, y}}$. Let $\mathscr{V}$ be an

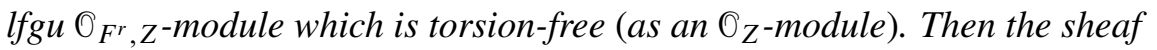

$$
\operatorname{Hom}_{\mathbb{O}_{F}, Z}\left(\mathscr{V}, \mathrm{O}_{Z}\right)
$$

is a constructible $\mathbb{E}_{p^{r} \text {-sheaf whose sections all have open support. The double-dual }}$ morphism

$$
\mathscr{V} \rightarrow \operatorname{Hom}_{\mathbb{F}_{p^{r}}}\left(\mathcal{H} m_{\mathscr{C}_{F}, Z}\left(\mathcal{V}, O_{Z}\right), \mathscr{O}_{Z}\right)
$$

is an isomorphism.

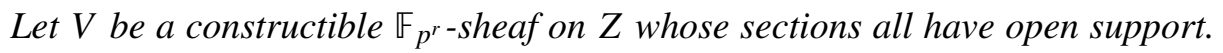
Then

$$
\operatorname{Hom}_{\mathbb{F}_{p^{r}}}\left(V, \mathrm{O}_{Z}\right)
$$

is a torsion-free lfgu $\mathbb{O}_{F^{r}}, Z$-module. The double-dual morphism

$$
V \rightarrow \operatorname{Hom}_{\mathbb{O}_{F}, Z}\left(\operatorname{Hom}_{\mathbb{F}_{p^{r}}}\left(V, \mathrm{O}_{Z}\right), \mathrm{O}_{Z}\right)
$$

is an isomorphism.

Proof. It is helpful at this point to assign labels to the functors in the RiemannHilbert correspondence. Let

$$
\mathbf{S}_{Z}(\cdot)=\operatorname{Hom}_{\mathscr{O}_{F}, Z}\left(\cdot, \mathfrak{O}_{Z}\right)
$$

and

$$
\mathbf{M}_{Z}(\cdot)=\operatorname{Hom}_{\mathbb{F}_{p} r}\left(\cdot, \mathrm{O}_{Z}\right)
$$




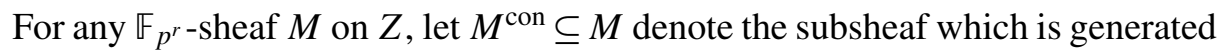
by the global sections of $M$. For any torsion-free unit $0_{F} r Z$-module $M$, let $\mathcal{M}^{\text {vec }} \subseteq$ $\mathcal{M}$ denote the subsheaf generated by $\Gamma(Z, M)^{\mathrm{vec}} \subseteq \Gamma(Z, \mathcal{M})$ (using notation from the previous subsection).

These two constructions satisfy a duality property under the Riemann-Hilbert correspondence. Suppose that $\phi$ is a global section of $\mathbf{M}_{Z}(M)$ (that is, an $\mathbb{F}_{p^{r-}}$ linear morphism from $M$ into $\mathrm{O}_{Z}$ ). If $\phi$ is contained in $\mathbf{M}_{Z}(M)^{\mathrm{vec}}$, then it is easy to see that the morphism of global sections,

$$
\Gamma(Z, M) \stackrel{\phi}{\longrightarrow} \Gamma\left(Z, O_{Z}\right)\left(=O_{Y, y}\right),
$$

must be trivial. The converse is also true. The set of morphisms $\phi$ for which (4.3.8) is trivial form a $K$-vector space (isomorphic to $\operatorname{Hom}_{\mathbb{F}_{p^{r}}}\left(M_{\mid \operatorname{Spec} K}, \mathrm{O}_{\operatorname{Spec} K}\right)$ ) which is contained in $\mathbf{M}_{Z}(M)^{\mathrm{vec}}$.

Thus, $\mathbf{M}_{Z}(M)^{\mathrm{vec}}$ is equal to the sheaf of morphisms $M \rightarrow \mathrm{O}_{Z}$ that kill $M^{\mathrm{con}}$.

Now suppose that $\mathcal{M}$ is a torsion-free finitely-generated unit $\mathcal{O}_{F^{r}, Z}$-module. It is clear that any $\mathrm{O}_{Z}$-morphism $\mathcal{M} \rightarrow \mathrm{O}_{Z}$ must kill $\mathcal{M}^{\mathrm{vec}}$. And, since $\mathcal{M} / \mathcal{M}^{\mathrm{vec}} \cong \mathrm{O}_{Z}^{\oplus d}$ for some $d$, it is easily seen that any section of $\mathscr{H} m_{\mathscr{O}_{F}, Z}\left(\mathcal{M}, O_{Z}\right)$ which kills $\mathcal{M}^{\text {vec }}$ can be extended to a global section. Therefore $\mathbf{S}_{Z}(\mathcal{M})^{\text {con }}$ is the subsheaf of $\mathbf{S}_{Z}(\mathcal{M})$ consisting of morphisms which kill $M^{\text {vec }}$.

We can now prove the local Riemann-Hilbert correspondence by building on Proposition 4.1.1 and Proposition 4.2.22. The duality discussed above implies that the sequence

$$
0 \rightarrow \mathbf{S}_{Z}\left(\mathscr{V} / \mathscr{V}^{\mathrm{vec}}\right) \rightarrow \mathbf{S}_{Z}(\mathscr{V}) \rightarrow \mathbf{S}_{Z}\left(\mathscr{V}^{\mathrm{vec}}\right) \rightarrow 0
$$

is exact. The sheaf $\mathbf{S}_{Z}\left(\mathscr{V} / \mathscr{V}^{\mathrm{vec}}\right)$ is constructible (in fact, constant), and the sheaf $\mathbf{S}_{Z}\left(\mathscr{V}^{\mathrm{vec}}\right)$ is constructible; therefore $\mathbf{S}_{Z}(\mathscr{V})$ is constructible. There is a diagram

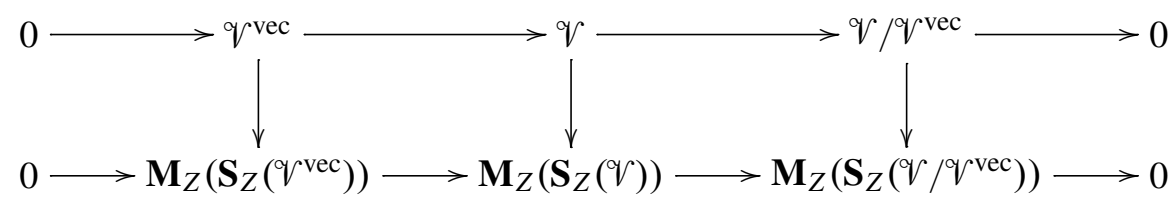

in which the vertical arrows are double-dual morphisms. The outer vertical arrows are isomorphisms by Proposition 4.1.1 and Proposition 4.2.22, and so the inner arrow is an isomorphism by the 5-lemma.

The second part of Theorem 4.3.1 follows similarly.

4.4. Local-to-global compatibility. The next two propositions show that the functors in the Riemann-Hilbert correspondence are compatible with localization. 
Proposition 4.4.1. Let $X$ be a smooth $k$-curve, and let $x$ be a closed point of $X$.

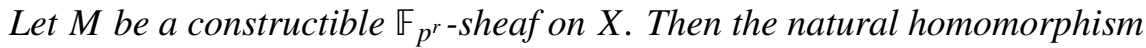

$$
\operatorname{Hom}_{\mathbb{F}_{p^{r}}}\left(M, \mathrm{O}_{X}\right)_{x} \rightarrow \operatorname{Hom}_{\mathbb{F}_{p^{r}}}\left(M_{(x)}, \mathrm{O}_{\mathrm{Spec} \mathrm{O}_{X, x}}\right)
$$

is an isomorphism.

Proof. By replacing $X$ with an appropriate étale neighborhood of $x$, we may make the following assumptions:

(1) $X$ is affine.

(2) There is a finite Galois morphism of curves

$$
\begin{aligned}
Z & \rightarrow X \\
z & \mapsto x,
\end{aligned}
$$

that is totally ramified at $x$ and étale elsewhere, and such that $M_{\mid Z \backslash\{z\}}$ is constant.

Let $R$ be the coordinate ring of the curve $X$, and let $S$ be the coordinate ring of the (affine) curve $Z \backslash\{z\}$. The automorphism group $G:=\operatorname{Aut}(Z / X)$ acts on $S$. Morphisms from the sheaf $M$ to the sheaf $O_{X}$ can be expressed as commutative diagrams

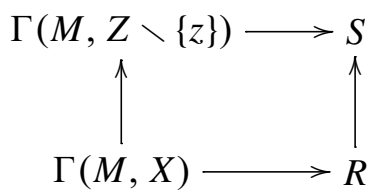

in which the top map is $G$-equivariant. Likewise morphisms from the sheaf $M_{(x)}$ to the sheaf $0_{\operatorname{Spec}} \mathscr{O}_{X, x}$ can be expressed as commutative diagrams

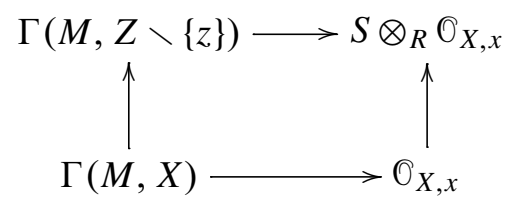

in which the top map is $G$-equivariant.

Suppose that we are given a diagram in the form of (4.4.4) above. Then since $\Gamma(M, Z \backslash\{z\})$ and $\Gamma(M, X)$ are both finite, there exists an étale subextension $B \subseteq O_{X, x}$ of $R$ such that the images of the top and bottom maps are contained in $S \otimes_{R} B$ and $B$, respectively. Thus we obtain a diagram

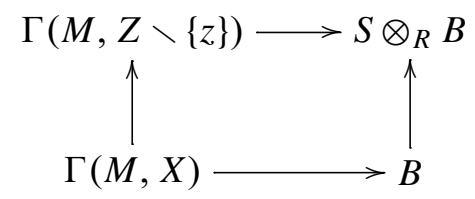


This argument shows that any morphism from $M_{(x)}$ to $\mathscr{O}_{\text {Spec } \mathscr{O}_{X, x}}$ can be extended to an affine étale neighborhood of $x$. This proves the proposition.

Proposition 4.4.6. Let $X$ be a smooth $k$-curve, and let $x$ be a closed point of $X$. Let $\mathcal{M}$ be an lfgu $\mathrm{O}_{F^{r}, X}$-module. Then the natural homomorphism

$$
\operatorname{Hom}_{\mathfrak{O}_{F}, X}\left(M, \mathscr{O}_{X}\right)_{x} \rightarrow \operatorname{Hom}_{\mathscr{O}_{X, x}\left[F^{r}\right]}\left(M_{x}, \mathscr{O}_{X, x}\right)
$$

is an isomorphism.

Proof. Replacing $X$ with an open subcurve if necessary, we may assume that $X$ is affine. Let $X=\operatorname{Spec} R$.

It suffices to show that any $\mathfrak{O}_{X, x}\left[F^{r}\right]$-module homomorphism $M_{x} \rightarrow \mathscr{O}_{X, x}$ can be extended to an étale neighborhood of $x$. Let $\phi: \mathcal{M}_{x} \rightarrow \mathcal{O}_{X, x}$ be such a homomorphism. Let

$$
\left\{m_{1}, \ldots, m_{\ell}\right\} \subseteq \Gamma(M, X)
$$

be a finite subset which generates $\mathcal{M}$ as an $\mathscr{O}_{F^{r}, X}$-module. Find an étale subextension $R \hookrightarrow R^{\prime}$ of $R \hookrightarrow \mathcal{O}_{X, x}$ which is large enough that $R^{\prime}$ contains the set

$$
\left\{\phi\left(\left(m_{1}\right)_{x}\right), \phi\left(\left(m_{2}\right)_{x}\right), \ldots, \phi\left(\left(m_{\ell}\right)_{x}\right)\right\} \subseteq \mathrm{O}_{X, x} .
$$

Then the homomorphism $\phi$ can be extended to a homomorphism from $M_{\mid\left(\operatorname{Spec} R^{\prime}\right)}$ to $\mathrm{O}_{\mathrm{Spec}} R^{\prime}$.

4.5. The proof of the global Riemann-Hilbert correspondence. We will now complete the proof of the Riemann-Hilbert correspondence on $Y$ (Theorem 2.3.11).

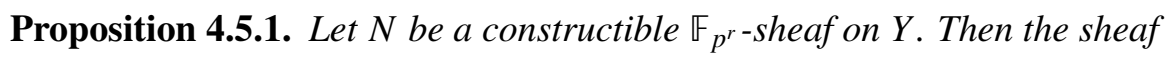

$$
\operatorname{Hom}_{\mathbb{F}_{p^{r}}}\left(N, \mathrm{O}_{Y}\right)
$$

is an lfgu $\mathrm{O}_{F^{r}, Y}$-module.

Proof. Let $\mathcal{N}^{\prime}$ denote the sheaf (4.5.2) above. Since $N$ is constructible, there exists an open subcurve $V \subseteq Y$ on which $N$ is locally constant of finite rank. The restriction $\mathcal{N}_{\mid V}^{\prime}$ is a coherent $\mathcal{O}_{V}$-module.

We can prove the desired properties of $\mathcal{N}^{\prime}$ from the analogous properties of its stalks. Note that Theorem 4.3.1 and Proposition 4.4.1 imply that each stalk $\mathcal{N}_{y}^{\prime}$, $y \in Y$, is a finitely-generated unit $\bigcirc_{Y, y}\left[F^{r}\right]$-module. Since the structural morphism $F_{Y}^{r *} \mathcal{N}^{\prime} \rightarrow \mathcal{N}^{\prime}$ is an isomorphism at each closed point $y \in Y$, it is globally an isomorphism. Moreover, since $\mathcal{N}_{\mid V}^{\prime}$ is a locally finitely-generated $\mathcal{O}_{F^{r}, V}$-module, and each stalk $\mathcal{N}_{y}^{\prime}$ at points $y$ outside of $V$ is a finitely-generated $O_{Y, y}\left[F^{r}\right]$-module, the sheaf $\mathcal{N}^{\prime}$ is a locally finitely-generated $\mathscr{O}_{F^{r}, Y}$-module.

It remains only to show that $\mathcal{N}^{\prime}$ is quasicoherent. Let $j: V \rightarrow Y$ denote the inclusion map. Consider the homomorphism

$$
\mathcal{N}^{\prime} \hookrightarrow j_{*}\left(\mathcal{N}_{\mid V}^{\prime}\right)
$$


The sheaf $j_{*}\left(\mathcal{N}_{\mid V}^{\prime}\right)$ is quasicoherent. The cokernel of (4.5.3) is easily seen to be a quasicoherent skyscraper sheaf. Therefore $\mathcal{N}^{\prime}$ is quasicoherent. This completes the proof.

Proposition 4.5.4. Let $\mathcal{N}$ be an lfgu $\mathcal{O}_{F^{r}, Y}$-module. Then the $\mathbb{F}_{p^{r} \text {-sheaf }}$

$$
\operatorname{Hom}_{\mathbb{O}_{F^{r}, Y}}\left(\mathcal{N}, \mathrm{O}_{Y}\right)
$$

is constructible.

Proof. Let $N^{\prime}$ denote sheaf (4.5.5) above. Theorem 4.3.1 and Proposition 4.4.6 imply that the stalks of $N^{\prime}$ are finite. To prove that $N^{\prime}$ is constructible, it suffices to show that there exists a nontrivial étale morphism $Z \rightarrow Y$ such that $N_{\mid Z}^{\prime}$ is constant.

Let $\eta$ be the generic point of $Y$. Let $L$ denote a separable closure of the residue field of $\eta$, and let

$$
\bar{\eta}: \operatorname{Spec} L \rightarrow Y
$$

denote the corresponding geometric generic point. The stalk $\mathcal{N}_{\bar{\eta}}$ is a finitelygenerated unit $L\left[F^{r}\right]$-module, which must be trivial.

Consider the finite subset

$$
\left(\mathcal{N}_{\bar{\eta}}\right)^{F^{r}} \subseteq \mathcal{N}_{\bar{\eta}} .
$$

There exists an étale open $U \rightarrow Y$ such that all elements of this subset can be realized as sections of $\mathcal{N}$ on $U$. Let $\mathscr{P} \subseteq \mathcal{N}_{\mid U}$ be the subsheaf generated by the $F^{r}$-invariant sections of $\Gamma(U, \mathcal{N})$. This subsheaf is a trivial $\mathscr{O}_{F^{r}, U}$-module.

The generic rank of $\mathscr{P}$ is the same as that of $\mathcal{N}_{\mid U}$. Since $\mathcal{N}_{\mid U}$ is coherent on a dense open subset of $U$ (by Proposition 2.1.7), it is easily seen that the sheaves $\mathcal{N}_{\mid U}$ and $\mathscr{P}$ agree on some nonempty open subset $U^{\prime} \subseteq U$.

Then, since $\mathcal{N}_{\mid U^{\prime}}$ is a trivial $\mathcal{O}_{F^{r}, U^{\prime}}$-module, the dual sheaf $N_{\mid U^{\prime}}^{\prime}$ is constant. This completes the proof.

Proof of Theorem 2.3.11. Propositions 4.5.1 and 4.5.4 assert that (2.3.12) is a constructible $\mathbb{F}_{p^{r}}$-sheaf and that (2.3.14) is an lfgu $O_{F^{r}, Y}$-module. The rest of the assertions in the theorem follow from Theorem 4.3.1 via Propositions 4.4.1 and 4.4.6.

\section{Acknowledgements}

This paper is the published version of my thesis from UC Berkeley. I gratefully acknowledge the help of my mentors, who have had extensive influence on the shape of the material here: Arthur Ogus (my thesis advisor), Martin Olsson, and Brian Conrad. I also thank Manuel Blickle, Igor Dolgachev, Kiran Kedlaya, Gennady Lyubeznik, Mark Kisin, Jacob Lurie, Brian Osserman, Richard Pink, Bjorn Poonen, Karen Smith, and Nicolas Stalder for helpful discussions. 


\section{References}

[Blickle 2004] M. Blickle, "The intersection homology D-module in finite characteristic", Math. Ann. 328:3 (2004), 425-450. MR 2005a:14005 Zbl 1065.14006

[Blickle 2008] M. Blickle, "Minimal $\gamma$-sheaves", Algebra Number Theory 2:3 (2008), 347-368. MR 2407119 Zbl 05529329

[Deligne and Katz 1973] P. Deligne and N. Katz (editors), Groupes de monodromie en géométrie algébrique, II: Séminaire de Géométrie Algébrique du Bois-Marie 1967-1969 (SGA 7 II), Lecture Notes in Math. 340, Springer, Berlin, 1973. MR 50 \#7135 Zbl 0275.14015

[Emerton and Kisin 2004] M. Emerton and M. Kisin, The Riemann-Hilbert correspondence for unit F-crystals, Astérisque 293, Soc. Math. France, Paris, 2004. MR 2005e:14027 Zbl 1056.14025

[Lyubeznik 1997] G. Lyubeznik, " $F$-modules: applications to local cohomology and $D$-modules in characteristic $p>$ 0”, J. Reine Angew. Math. 491 (1997), 65-130. MR 99c:13005 Zbl 0904.13003

[Pink 2000] R. Pink, "Euler-Poincaré formula in equal characteristic under ordinariness assumptions”, Manuscripta Math. 102:1 (2000), 1-24. MR 2001j:11048 Zbl 1010.11034

[Raynaud 1995] M. Raynaud, “Caractéristique d'Euler-Poincaré d'un faisceau et cohomologie des variétés abéliennes”, pp. 129-147, Exp. No. 286 in Séminaire Bourbaki, Vol. 9, Soc. Math. France, Paris, 1995. MR 1608794

Communicated by Hélène Esnault

Received 2009-03-21 Revised 2009-10-21 Accepted 2009-11-23

carlmi@umich.edu

Department of Mathematics, 530 Church Street, University of Michigan, Ann Arbor, MI 48109, United States http://www.umich.edu/ carlmi/ 